\title{
ESTIMACIÓN Y ANÁLISIS DE LA INCIDENCIA DE VIH EN POBLACIÓN ADULTA DEL PERÚ: RESULTADOS DE LA APLICACIÓN DEL MODELO MATEMÁTICO MOT
}

\author{
Jorge O. Alarcón 1,a, Mónica Pun²,a, César Gutiérrez,a, Álvaro Whittembury,a, Romina Tejada ${ }^{1, b}$, \\ Luis Suárez ${ }^{2, a}$, Gustavo Rosell ${ }^{3, b}$, Annick Bórquez ${ }^{4, c}$, Paloma Cuchi5,a
}

\section{RESUMEN}

Objetivos. Estimar la incidencia de VIH en la población adulta del Perú, 2010, y analizar su distribución según comportamientos de riesgo. Materiales y métodos. Se aplicó el modelo de ONUSIDA según modos de transmisión (MoT). Los datos fueron obtenidos de la revisión detallada de 59 documentos (1984 - 2008). También se analizó bases de datos nacionales para obtener datos específicos. La selección final de los datos fue validada por el grupo técnico y un grupo de expertos. Después de la consulta con expertos, se corrigieron los valores y se realizó un análisis de incertidumbre. El modelo fue ajustado a la prevalencia nacional del 2009 (0,45\%). Resultados. La incidencia estimada para el 2010 fue de 0,03\%, (4346 nuevas infecciones). El 84\% de nuevas infecciones se concentra en grupos de mayor riesgo: hombres que tienen sexo con hombres (55\%) y personas que tienen sexo casual heterosexual $(6,2 \%)$. El 16\% restante corresponde a la población heterosexual de bajo riesgo. La transmisión heterosexual es el $43 \%$ de nuevos casos, y dentro de estos el $18 \%$ corresponde a parejas femeninas de sujetos de alto riesgo. Solo $2,2 \%$ de casos está relacionado al trabajo sexual femenino y 1,0\% a usuarios de drogas inyectables. Conclusiones. El modelo proporciona una estimación de la incidencia y su distribución entre los grupos de riesgo según el modo de transmisión, consistente con los reportes de casos de VIH. El modelo permite crear escenarios para ayudar a la toma de decisiones y formulación de políticas, así como para vigilancia y planificación de la prevención y control.

Palabras clave: VIH; Modelos matemáticos; Técnicas de estimación; Incidencia (fuente: DeCS BIREME).

\section{ESTIMATION AND ANALYSIS OF HIV INCIDENCE IN THE ADULT POPULATION IN PERU: RESULTS OF THE APPLICATION OF THE MOT MATHEMATIC MODEL}

\begin{abstract}
Objectives. To estimate HIV incidence in the adult population of Peru, 2010, and analyze its distribution based on risk behavior. Materials and methods. The UNAIDS model was applied based on the modes of transmission (MoT). The information was obtained from the review detailed in 59 documents $(1984-2008)$. National databases were also analyzed to obtain specific data. Final selection of data was validated by the technical group and a group of experts. After consultation with experts, values were corrected and an uncertainty analysis was conducted. The model was adjusted to 2009 national prevalence $(0.45 \%)$. Results. Incidence estimated for 2010 was $0.03 \%$, (4346 new infections). $84 \%$ of new infections concentrate on higher risk groups: men who have sex with men (55\%) and people who have casual sex with heterosexuals (6.2\%). The remaining $16 \%$ corresponds to low-risk heterosexual population. Heterosexual transmission accounts for $43 \%$ of new cases, $18 \%$ of which corresponds to female partners of high risk individuals. Only $2.2 \%$ of cases is related to female sexual work and $1.0 \%$ to injection drug users. Conclusions. The model provides an estimation of the incidence and its distribution among risk groups according to the mode of transmission, consistent with the HIV case reporting. The model creates scenarios to help decision making and policy formulation, as well as surveillance and planning of prevention and control.
\end{abstract}

Key words: HIV; Mathematical models; Estimation techniques; Incidence (source: MeSH NLM).

\footnotetext{
Sección de Epidemiología, Instituto de Medicina Tropical “Daniel A. Carrión”; Universidad Nacional Mayor de San Marcos. Lima, Perú.

Dirección General de Epidemiología, Ministerio de Salud. Lima, Perú.

Dirección Regional de Salud San Martín. San Martín, Perú.

Imperial College London. Londres, Reino Unido.

Programa Conjunto de las Naciones Unidas sobre el VIH/SIDA (ONUSIDA). Ginebra, Suiza.

Médico epidemiólogo; ${ }^{\mathrm{b}}$ médico cirujano; ${ }^{\mathrm{c}}$ médico epidemiólogo especialista en Microbiología

Recibido: 14-05-12 Aprobado: 14-11-12

* El informe preliminar de este estudio fue presentado en la XVIII Conferencia Internacional sobre SIDA, Viena, julio de 2010.
}

Citar como: Alarcón J, Pun M, Gutiérrez C, Whittembury A, Tejada R, Suárez L, Rosell G, Bórquez A, Cuchi P. Estimación y análisis de la incidencia de VIH en población adulta del Perú: resultados de la aplicación del modelo matemático MoT. Rev Peru Med Exp Salud Publica. 2012;29(4):452-60. 


\section{INTRODUCCIÓN}

En el Perú se cuenta con información sobre la prevalencia de VIH/SIDA, particularmente de los grupos de mayor riesgo; sin embargo, hay pocos estudios acerca de su incidencia por las dificultades para estimarla (particularidades de la historia natural, costo y tiempo de dichos estudios).

La incidencia es el mejor indicador para conocer el curso de la epidemia, identificar los puntos de intervención y prever los recursos para una prevención efectiva. Entre las alternativas para estimar la incidencia ${ }^{(1,2)}$ lo que más se ha usado es la elaboración de modelos matemáticos, siendo necesario contar con información confiable sobre prevalencia y asumir supuestos de la supervivencia y tasas de transmisión ${ }^{(3-5)}$. Sin embargo, estas estimaciones no proporcionan información acerca de los comportamientos de riesgo y, por ende, no permiten dirigir los esfuerzos de prevención hacia los grupos más vulnerables. Frente a esta necesidad, el año 2001 el grupo de referencia en estimaciones, modelamiento y proyecciones de ONUSIDA desarrolló el modelo de modos de transmisión (MoT) ${ }^{(6)}$ que permite estimar los casos incidentes de $\mathrm{VIH}$ del año próximo según diferentes comportamientos de riesgo a partir de datos de prevalencia, siendo sus ventajas el ser relativamente sencillo de aplicar y demandar menor cantidad de datos comparado con otros. La información obtenida (nuevas infecciones por comportamiento de riesgo a corto plazo) permite formular un mensaje claro y conciso para la prevención. El modelo se ha aplicado en varios países, está disponible junto con el manual para su aplicación en el portal de ONUSIDA (http:/www.unaids.org) y ha sido descrito en otros artículos $(7,8)$.
En Perú, las primeras predicciones de la tendencia de la epidemia del VIH fueron hechas el año 2000 , utilizando el programa EPIMODEL (6); en los últimos años se han empleado otros modelos como Spectrum y EPP. Esto permitió, en su momento, estimar el número de personas viviendo con el $\mathrm{VIH}$, número de casos incidentes anuales, número de personas que requerirán tratamiento antirretroviral de gran actividad (TARGA) y la magnitud de la mortalidad por SIDA. Los datos empleados en estas aplicaciones fueron obtenidos de la vigilancia centinela, notificación de casos de VIH/ SIDA, atenciones en los centros de referencia de ETS (CERETS) y datos poblacionales del Instituto Nacional de Estadística e Informática (INEI), entre otras fuentes.

En este trabajo se estima la proporción de nuevas infecciones a corto plazo por modo de transmisión, obtenido a través de la aplicación del MoT al Perú, usando como fuentes la información generada por el sistema de vigilancia, los reportes de atenciones de los servicios hospitalarios y ambulatorios y por los estudios realizados entre 1983 y 2008.

\section{MATERIALES Y MÉTODOS}

\section{MODELO DE MODOS DE TRANSMISIÓN}

El MoT es un modelo probabilístico estático basado en el teorema de Bernoulli, que calcula la proporción de nuevas infecciones por comportamiento de riesgo a corto plazo a partir del número promedio de contactos y de la prevalencia de $\mathrm{VIH}$ de estos contactos. Requiere identificar grupos con comportamientos de riesgo específicos y conocer su tamaño, prevalencia actual de VIH e ITS, frecuencia de exposición y protección, y la

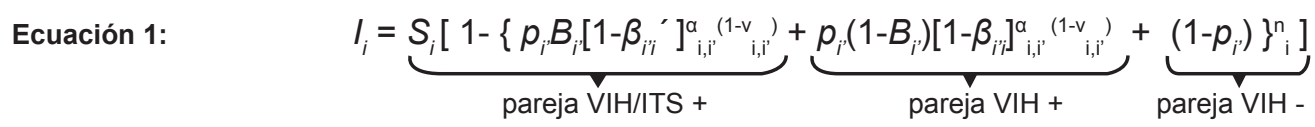

I: incidencia en el grupo i; S: número de individuos susceptibles en este grupo; p.: prevalencia de VIH de la pareja; B: prevalencia de ITS de la pareja; $\beta$ : probabilidad de transmisión de VIH; $\beta$ ': probabilidad de transmisión de VIH en presencia de una ITS; $\alpha$ : número de relaciones sexuales por pareja; v: proporción de relaciones sexuales protegidas por uso de condón; n: número de parejas.

Ecuación 2:

$$
I_{i}=\underbrace{S_{i}\left[1-\left\{p_{i}\left[1-\beta_{i j}\right]_{i, i}^{\alpha}\left(1-\mathrm{v}_{\mathrm{i}, \mathrm{i}}\right)\right.\right.}_{\text {contacto } \mathrm{VIH}+}+\underbrace{\left.\left.\left(1-p_{i}\right)\right\}_{\mathrm{i}}^{\mathrm{n}}\right]}_{\text {contacto } \mathrm{VIH}-}
$$

I: incidencia en el grupo i; S: número de individuos susceptibles en este grupo; $p$ ': prevalencia de VIH en compañeros que comparten jeringas; $\beta$ : probabilidad de transmisión de $\mathrm{VIH}$; $\alpha$ : contactos por compañeros; $v$ : uso de jeringas estériles; $n$ : número de compañeros.

Figura 1. Ecuación estándar del modelo que calcula la incidencia de nuevas infecciones por VIH adquiridas a través de contacto sexual entre las personas con una conducta de riesgo específica y sus parejas (ecuación 1) o por vía parenteral (ecuación 2). 
probabilidad específica de transmisión según el modo de exposición.

La estimación de la incidencia de VIH por transmisión sexual y parenteral (en UDI) en cada grupo $i$ se obtiene de las ecuaciones 1 y 2 (Figura 1). Los grupos incluidos en el modelo y los contactos entre estos considerados por el modelo están en la Anexo 1 (disponible solo en la versión electrónica). La transmisión por inyecciones médicas y transfusiones sanguíneas se calcula con la misma ecuación de la transmisión parenteral en UDI, pero se considera que los contactos provienen de la población total y no de un solo grupo.

El número de nuevas infecciones por transmisión sexual en un grupo es igual al número de individuos susceptibles en ese grupo por 1 , menos la probabilidad de no infectarse (1- $\beta$ ) después de $\alpha$ actos sexuales de los cuales (1-v) no son protegidos, con cada una de las parejas que pueden ser: VIH e ITS positivas, o VIH positivas pero ITS negativas o VIH negativas. Estos tres tipos de parejas suman: $\mathrm{pB}+\mathrm{p}(1-B)+(1-p)=1$.

El efecto de las ITS se incorpora en la probabilidad de transmitir la infección por VIH, pero no en la de adquirirla, suponiendo que el riesgo de transmitir VIH por personas ITS positivas es cuatro veces mayor ${ }^{(9,10)}$.

El efecto de la circuncisión se incorpora modificando directamente la probabilidad de transmisión de mujer a hombre por $60 \%$ por la proporción de hombres circuncidados ${ }^{(11)}$. Esta probabilidad se aplica a todos los actos sexuales entre hombres y mujeres, por ende se supone que la proporción de circuncidados es igual en todos los grupos de hombres del modelo.

Los grupos considerados en el modelo son usuarios de drogas inyectables (UDI) y sus parejas sexuales, trabajadoras sexuales (TS), sus clientes y las parejas sexuales de estos, los HSH y sus parejas sexuales estables mujeres, las personas que tienen sexo casual heterosexual y sus parejas estables, las personas que tienen sexo heterosexual de bajo riesgo (monógamos) y las personas sin riesgo de infectarse por vía sexual o compartir jeringas. Se considera que cada grupo únicamente se infecta por un tipo de exposición (Anexo 1). Los UDI por ejemplo, solo se infectan por compartir jeringas, aunque también tengan parejas sexuales.

\section{FUENTES DE DATOS}

Los datos fueron recolectados de los archivos proporcionados por la Dirección General de Epidemiología (DGE) y de la búsqueda sistemática en PubMed y LILACS, empleando los términos VIH, ETS/ITS, Perú y los relativos a comportamientos de riesgo. Se revisó 59 documentos (1984 - 2008), extrayendo los datos por una ficha ad hoc: población estudiada, periodo y ámbito de estudio, vía de transmisión, nivel de riesgo e indicadores utilizados como prevalencia e incidencia. También se analizaron para obtener datos específicos la ENDES 2004-2006 ${ }^{(12)}$ y $2008{ }^{(13)}$, el Censo Nacional $2007^{(14)}$ y la Encuesta Nacional de Consumo de Drogas $2006{ }^{(15)}$. La selección final de datos fue validada por el grupo técnico y un grupo de expertos.

\section{SELECCIÓN DE LOS DATOS}

El modelo se ajustó a la prevalencia nacional del 2009 $(0,45 \%)^{(16)}$ y a una población de 14514191 personas entre 15 y 49 años ${ }^{(14)}$. Para los casos de las parejas femeninas de $\mathrm{HSH}$, UDI, clientes de TS y personas que practican sexo casual heterosexual, se asignó una pareja sexual por año ${ }^{(12)}, 77$ actos sexuales por pareja por año ${ }^{(17)}$ y $7,45 \%$ de uso de condón ${ }^{(12)}$. Se siguieron los siguientes criterios para la inclusión de datos:

Hombres que tienen sexo con hombres (HSH) y sus parejas: los HSH representaban el $6 \%$ de la población masculina según los estudios de Cárcamo et al. (comunicación personal antes de la publicación del estudio PREVEN) ${ }^{(18)}$ y Cáceres et al ${ }^{(19)}$, asignándoles una prevalencia de $5,16 \%{ }^{(14,20,21)}$, ajustada al número de muertes y subregistro. No se empleó los datos de Baral et al. ${ }^{(22)}(12,2 \%)$ debido al sesgo de selección en el metanálisis. La prevalencia de ITS se estimó en $6 \%{ }^{(18)}$. No se consideraron los datos de Lama et al. ${ }^{(22)}$ por ser una población HSH de alto riesgo. Se asumió 2,7 parejas por año ${ }^{(18,23)}$ con doce actos de exposición por pareja por año ${ }^{(18)}$ y $70 \%$ de actos protegidos ${ }^{(23,24)}$.

Se consideró que el porcentaje de parejas femeninas de HSH fue 1,14\% de la población femenina (el 19,0\% de HSH reporta pareja femenina estable $\left.{ }^{(25)}\right)$. Se asignó una prevalencia de $1,6 \%$ (multiplicación del valor de vigilancia centinela $(0,23 \%){ }^{(26)}$ y un OR de siete ${ }^{(27)}$.

Usuarios de drogas intravenosas (UDI) y sus parejas: la prevalencia de vida de drogas intravenosas en 2006 fue $0,03 \%$ en varones y ningún caso en mujeres ${ }^{(15)}$. No existen datos nacionales de prevalencia de $\mathrm{VIH}$ para este grupo por lo que se usó el reporte de Mathers et al. ${ }^{(28)}$ de $13 \%$. Se estimó una prevalencia de ITS en $3,15 \%{ }^{(29)}$ (prevalencia de sífilis de $1,5 \%$ ) y el OR de 2,1 para la presencia de cualquier ITS ${ }^{(30)}$. Se consideraron dos parejas de inyección por año (31) y 95 actos de intercambio de agujas con cada pareja de inyección por año ${ }^{(32)}$ y un total de 190 actos de exposición por año ${ }^{(33)}$. 
Bajo el supuesto que los varones UDI tienen el mismo número de parejas sexuales femeninas que la población masculina general $(52,9 \%)^{(14)}$, la proporción de parejas sería de $0,02 \%$ de la población general y la prevalencia de $\mathrm{VIH} \mathrm{2,5 \%}{ }^{(27)}$.

Trabajadoras sexuales (TS): las mujeres TS se estimaron en $0,5 \%{ }^{(34)}$ de la población femenina. La prevalencia de VIH se estimó en $0,9 \%{ }^{(35)}$ y de ITS $15,0 \%{ }^{(18,36)}$. Se estimó que cada TS en promedio tiene 843 parejas sexuales por año ${ }^{(18)}$ y se asumió un acto sexual por cliente al año, con $80,6 \%$ de actos sexuales protegidos ${ }^{(37-39)}$.

Clientes de TS y sus parejas: el porcentaje de clientes de TS se calculó en 15,8\% (18,27,38). La prevalencia de VIH se estimó en $0,76 \%$ (prevalencia de vigilancia centinela ${ }^{(26)}(0,27 \%)$ multiplicada por OR de $\left.2,8{ }^{(27)}\right)$. Del mismo modo, se estimó la prevalencia de ITS en $3,3 \%{ }^{(29,40)}$. La estimación resultante fue compatible con datos previamente descritos ${ }^{(18,38)}$. El número promedio de parejas al año de los clientes de TS se calculó en 26, según la fórmula: (número de TS x número de actos sexuales)/número de clientes; se mantuvo el supuesto de un acto sexual por pareja por año y de un $80,6 \%$ de actos sexuales protegidos.

Se asumió que la proporción de parejas de los clientes de TS es $8,4 \%$ de la población femenina, suponiendo que los clientes de TS tienen parejas en una proporción similar a la población masculina ${ }^{(14)}$, y que la prevalencia de $\mathrm{VIH}$ es la misma que la informada en la vigilancia centinela ${ }^{(26)}$ multiplicado por un OR de 2,8 ${ }^{(27)}$.

Personas que tienen sexo casual heterosexual $(\mathrm{SCH})$ : se consideró $\mathrm{SCH}$ cuando se tiene relaciones sexuales con una persona con la que no se cohabita, no es regular o tiene múltiples parejas ${ }^{(41)}$. La proporción de hombres en este grupo fue de $21,0 \%{ }^{(13)} y$ de mujeres de $11,0 \%{ }^{(12,31)}$. Se les asignó una prevalencia de VIH similar a la de la población general ${ }^{(26)}$ y para ITS de $12,0 \%{ }^{(42)}$. Se consideró que tienen 1,5 parejas sexuales por año (promedio de hombres y mujeres) ${ }^{(12,13)}, 39$ actos sexuales por pareja por año (50\% de actos sexuales con una pareja estable) ${ }^{(28)}$ y uso de condón del $27,2 \%{ }^{(12)}$.

Las parejas varones representaron el 5,8\% y las parejas mujeres el $11,2 \%$ de la población general, valores obtenidos de multiplicar el número de mujeres y hombres que practican $\mathrm{SCH}$ por 0,54 y 0,53 respectivamente (porcentaje de unidos) ${ }^{(14)}$. Se les asignó una prevalencia de $\mathrm{VIH}$ igual a la de sus parejas ${ }^{(26)}$.

Personas sin riesgo: los varones sin riesgo de $\mathrm{VIH}$ representaban el $29,7 \%{ }^{(13)}$ y las mujeres $33,0 \%{ }^{(12)}$, equivalente al porcentaje de hombres no unidos y mujeres que nunca han tenido relaciones sexuales o que han tenido relaciones sexuales hace un año o más. Heterosexuales de bajo riesgo: personas casadas o con pareja estable no incluidas en los otros grupos; representó el $26,8 \%$ de hombres y $37,1 \%$ de mujeres. Se asignó una prevalencia de $\mathrm{VIH}$ de $0,25 \%{ }^{(26)}$ y de ITS de $9,0 \%{ }^{(18)}$.

Transfusiones sanguíneas: se asumió que el 0,5\% de la población femenina y masculina han recibido en algún momento de su vida una transfusión, dado que la prevalencia de hemofilia y otras enfermedades que requieran transfusiones es muy baja. Se asignó una prevalencia de $0,25 \%{ }^{(26)}$ y se consideró que el $100 \%$ de unidades de sangre son tamizadas eficientemente para VIH ${ }^{(43)}$.

Datos adicionales: finalmente, se estableció que el 5,7\% de hombres adultos es circuncidado ${ }^{(44)}$. Se asumió que toda la población en algún momento de su vida ha recibido alguna inyección médica, por lo que estarían incluidos en los otros grupos, con una prevalencia de $\mathrm{VIH}$ igual que la población general ${ }^{(26)}$ y una protección del $95 \%$.

\section{VALIDACIÓN CON EXPERTOS NACIONALES}

Se realizó una reunión para la validación interna del modelo a la que se invitó a expertos en el tema, (MINSA, universidades y sociedad civil). Se presentaron los fundamentos del modelo y los datos ingresados. Se discutió la confiabilidad de dichos datos, el uso de otras fuentes y se entregó un formulario con la relación de los datos y se pidió que en caso necesario proporcionaran referencias para obtener una mejor estimación.

\section{ANÁLISIS DE INCERTIDUMBRE}

Tomando en cuenta que el MoT requiere una cantidad de datos importante para su parametrización, es difícil contar con toda la información necesaria. Por ello, ciertos parámetros se basan en inferencias, en datos de otros países o en supuestos. Además, aunque se cuente con información nacional, los estimados inevitablemente tienen cierta incertidumbre. Se asignó un porcentaje de variación a cada parámetro (0 al 100\%). Todos los parámetros varían, excepto el número de parejas en el grupo de bajo riesgo y en las parejas de miembros del grupo de riesgo (por definición es uno). El tamaño de los grupos que son parejas de miembros de grupos de riesgo no varía directamente sino que se le asigna una proporción fija del tamaño del grupo de riesgo.

Se hizo un muestreo de tipo Monte Carlo, de manera que cada parámetro variara simultánea, aleatoria e independientemente a cada corrida del modelo. Para que la población total sumara al $100 \%$ y la prevalencia 
total correspondiera a la estimada en el modelo original, el tamaño y la prevalencia del grupo de bajo riesgo se calcularon como el remanente de los otros grupos. Para seleccionar sets de parámetros que resultaran en una estimación de la incidencia plausible, se aplicó como filtro el rango de nuevas infecciones estimadas con el modelo Spectrum de ONUSIDA. Se repitió el proceso hasta obtener 1000 corridas que produjeran un número de nuevas infecciones dentro de este rango. El rango de incertidumbre obtenido corresponde a los percentiles 2,5 y 97,5 del número total de nuevas infecciones obtenidas en las 1000 corridas.

\section{RESULTADOS}

Los resultados de la estimación se presentan en la Tabla 1. La incidencia de VIH en la población adulta entre 15 y 49 años para el 2010 se estima en 0,03\% (4346 nuevas infecciones). Sin considerar a los UDI y a sus parejas, los grupos de mayor riesgo son los $\mathrm{HSH}$, las parejas femeninas de los HSH y las TS. La distribución proporcional de estas infecciones según grupo de riesgo se presenta en la penúltima columna de la Tabla, observando que $84,0 \%$ de las nuevas infecciones se concentra en población de mayor riesgo, destacando los $\mathrm{HSH}(55,0 \%)$; le siguen en importancia el grupo de personas que tienen $\mathrm{SCH}(6,2 \%)$. El 16,0\% restante corresponde a nuevas infecciones en la población heterosexual de bajo riesgo.

La transmisión heterosexual ocurre en el 43,0\% de nuevos casos; $18,0 \%$ corresponde a parejas femeninas de sujetos de alto riesgo (clientes de TS, HSH, UDI y $\mathrm{SCH}), 16,0 \%$ en población femenina y masculina de bajo riesgo, $6,2 \%$ en personas que tienen $\mathrm{SCH}$ y solo $2,2 \%$ en grupos vinculados al trabajo sexual femenino (TS y sus clientes). Finalmente los UDI contribuyen con el $1,0 \%$ de nuevas infecciones.

Tabla 1. Hoja de cálculo MoT parametrizada con datos de Perú.

\begin{tabular}{|c|c|c|c|c|c|c|c|c|c|c|c|c|c|c|}
\hline \multirow{2}{*}{$\begin{array}{l}\text { Comporta- } \\
\text { miento de } \\
\text { riesgo }\end{array}$} & \multicolumn{2}{|c|}{$\begin{array}{l}\text { Población con } \\
\text { conductas de } \\
\text { riesgo }(\%)\end{array}$} & \multirow[t]{2}{*}{ N. ${ }^{\circ *}$} & \multirow[t]{2}{*}{$\begin{array}{l}\text { Prev. } \\
\text { VIH (\%) }\end{array}$} & \multirow[t]{2}{*}{$\begin{array}{l}\text { Número } \\
\text { VIH + }\end{array}$} & \multirow[t]{2}{*}{$\begin{array}{l}\text { Prev. } \\
\text { ITS (\%) }\end{array}$} & \multirow[t]{2}{*}{$\begin{array}{c}\text { Parejas } \\
\text { por año } \\
\left(\mathbf{N}^{\circ}\right)\end{array}$} & \multirow{2}{*}{$\begin{array}{c}\text { Actos de } \\
\text { exposición } \\
\text { por pareja } \\
\text { y año } \\
\left(N^{\circ}\right)\end{array}$} & \multirow{2}{*}{$\begin{array}{l}\text { Actos } \\
\text { prote- } \\
\text { gidos } \\
(\%)\end{array}$} & \multicolumn{2}{|c|}{$\begin{array}{l}\text { Probabilidad } \\
\text { de transmisión } \\
\text { por exposición } \\
\text { con riesgo }\end{array}$} & \multirow{2}{*}{$\begin{array}{l}\text { Inciden- } \\
\text { cia }\end{array}$} & \multirow{2}{*}{$\begin{array}{l}\text { Distri- } \\
\text { bución } \\
\% \text { de la } \\
\text { inciden- } \\
\text { cia }\end{array}$} & \multirow{2}{*}{$\begin{array}{l}\text { Inciden- } \\
\text { cia por } \\
100000\end{array}$} \\
\hline & Hombres & Mujeres & & & & & & & & $\begin{array}{l}\text { con } \\
\text { ITS }\end{array}$ & Sin ITS & & & \\
\hline $\begin{array}{l}\text { Uso drogas } \\
\text { inyectables } \\
\text { (UDI) }\end{array}$ & 0,033 & 0,00 & 2402 & 13,00 & 312 & 3,15 & 1 & 190 & 80,00 & & 0,0100 & 86 & 1,98 & 3590 \\
\hline Parejas UDI & 0,00 & 0,017 & 1241 & 2,50 & 31 & NA & 1 & 77 & 7,45 & 0,002 & 0,0008 & 9 & 0,22 & 762 \\
\hline $\begin{array}{l}\text { Trabajadoras } \\
\text { sexuales (TS) }\end{array}$ & & 0,49 & 35560 & 0,90 & 320 & 15,00 & 843 & 1 & 80,60 & 0,002 & 0,0008 & 39 & 0,89 & 108 \\
\hline Clientes TS & 15,80 & & 1146621 & 0,76 & 8714 & 3,30 & 26 & 1 & 80,60 & 0,002 & 0,0008 & 58 & 1,33 & 5 \\
\hline $\begin{array}{l}\text { Parejas de } \\
\text { clientes TS }\end{array}$ & & 8,358 & 606563 & 0,64 & 3882 & NA & 1 & 77 & 7,45 & 0,003 & 0,0008 & 276 & 6,36 & 46 \\
\hline $\begin{array}{l}\text { Hombres que } \\
\text { tienen sexo } \\
\text { con hombres } \\
\text { (HSH) }\end{array}$ & 6,00 & & 435426 & 5,16 & 22468 & 6,00 & 2,7 & 12 & 70,00 & 0,040 & 0,0100 & 2,389 & 54,97 & 549 \\
\hline $\begin{array}{l}\text { Parejas } \\
\text { femeninas de } \\
\text { HSH }\end{array}$ & & 1,140 & 82731 & 1,61 & 1332 & NA & 1 & 77 & 7,45 & 0,003 & 0,0008 & 270 & 6,22 & 327 \\
\hline $\begin{array}{l}\text { Sexo casual } \\
\text { heterosexual } \\
(\mathrm{SCH})\end{array}$ & 21,02 & 11,00 & 2323722 & 0,27 & 6274 & 12,00 & 1,5 & 39 & 27,26 & 0,002 & 0,0008 & 274 & 6,29 & 12 \\
\hline Parejas SCH & 5,87 & 11,12 & 1233240 & 0,27 & 3330 & NA & 1 & 77 & 7,45 & 0,003 & 0,0008 & 241 & 5,54 & 20 \\
\hline $\begin{array}{l}\text { Sexo hete- } \\
\text { rosexual de } \\
\text { bajo riesgo }\end{array}$ & 21,54 & 34,88 & 4094310 & 0,25 & 10236 & 9,00 & 1 & 77 & 7,45 & 0,003 & 0,0008 & 694 & 15,97 & 17 \\
\hline $\begin{array}{l}\text { Transfusiones } \\
\text { sanguíneas** }\end{array}$ & 0,50 & 0,50 & 72571 & 0,25 & & NA & 1 & 1 & 100,00 & & 0,9000 & 0 & 0,00 & 0 \\
\hline $\begin{array}{l}\text { Población } \\
\text { adulta total }\end{array}$ & 100 & 100 & 14514191 & 0,45 & 66004 & & & & & & & 4346 & & 30 \\
\hline
\end{tabular}

*: Estimado de personas con comportamiento de riesgo ** No se consideran conductas excluyentes; Prev: prevalencia; NA: no aplica. 


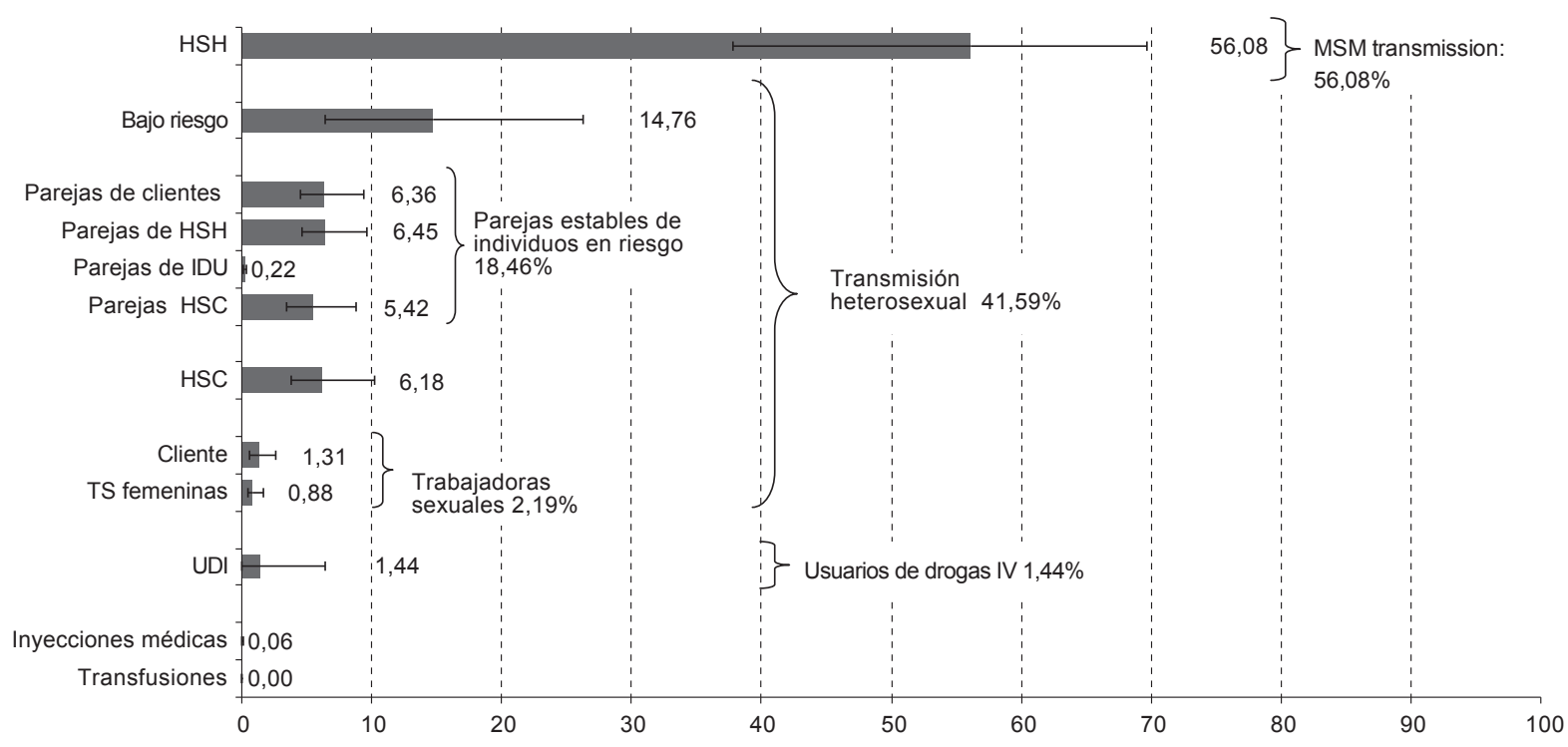

Figura 2. Límites de incertidumbre alrededor de la distribución de nuevas infecciones por VIH según modo de transmisión en Perú. $\mathrm{HSH}$ : hombres que tienen sexo con hombres; HSC: heterosexuales que realizan sexo casual; UDI: usuarios de drogas endovenosas.

Los valores y límites de incertidumbre para cada grupo están en la Figura 2. Pese a la amplitud de los intervalos, es claro el predominio de los HSH.

\section{DISCUSIÓN}

La incidencia estimada por el modelo $(0,03 \%)$ es consistente con una epidemia concentrada, como la que afecta al país y cercana a los datos de notificación nacional. Sin tomar en cuenta los UDI y sus parejas, la población de $\mathrm{HSH}$ es la de mayor incidencia y representa el $55 \%$ (intervalo de incertidumbre: $38-70 \%$ ) del total de casos nuevos estimados, no obstante ser el $6 \%$ de la población adulta.

Los otros grupos de incidencia elevada contribuyen en menor proporción al total de casos nuevos, por tener menor incidencia que los $\mathrm{HSH}$ y representan una proporción pequeña de la población. Los UDI, que tiene la incidencia más alta, constituirían el $1 \%$ de los casos nuevos. En el Perú casi no se reportan casos de VIH con este hábito, y el hábito mismo está muy poco extendido. Es posible incluso, que este valor sea consecuencia de una sobrestimación tanto de la proporción de usuarios de este tipo de drogas como de la probabilidad de transmisión en el país.

En el caso de las TS ocurre algo similar. Tienen una incidencia elevada, aunque bastante menor que los $\mathrm{HSH}$, pero solo representan el $0,8 \%$ de los casos nuevos estimados. Esto es consistente con el hecho que los estudios realizados en este grupo encuentran que la prevalencia es baja y un elevado uso de condón, probablemente debido a las múltiples intervenciones de prevención. Por el momento, este resultado debilita el argumento de considerar a las TS como población puente para la transmisión del $\mathrm{VIH}$ de $\mathrm{HSH}$ a personas heterosexuales, pudiendo ser tal vez la población bisexual la que este desempeñando dicho papel en el país. Sin embargo, hay que tomar en cuenta que el modelo no considera el contacto entre TS y HSH y que algunos datos de los estudios de prevalencia en esta población son incompletos. No hay que perder de vista, según el modelo, que la incidencia en este grupo es alta (casi cinco veces superior a la de la población de bajo riesgo), hecho que puede ser importante para el futuro desarrollo de la epidemia.

Es diferente la situación en la población heterosexual de bajo riesgo, que con una incidencia mucho menor contribuye con el $16 \%$ de los casos nuevos, puesto que representan casi el $30 \%$ de la población, que contrasta con el $6 \%$ que se atribuye a la población de $\mathrm{HSH}$ pero que por su elevada incidencia contribuye significativamente al total de casos incidentes estimados para el año 2010.

En suma, el modelo proporciona una estimación de la incidencia global y específica por grupos de riesgo aceptable, que será de mucha utilidad para la priorización de las actividades preventivas, ofreciendo sustento a diversas estrategias de intervención. Por ejemplo, si se toma en cuenta que casi la mitad de los casos nuevos corresponden a HSH, que a su vez 
se estiman son el $6 \%$ de la población adulta de 15 a 49 años, es razonable pensar que las acciones de prevención en este grupo pueden ser más eficientes y producir un impacto importante en el curso de la epidemia en el país. Si a esto se suma el hecho de que el $18 \%$ de nuevas infecciones se presentarán en parejas estables de grupos de riesgo, es recomendable extender y fortalecer las actividades preventivas a estas parejas y a la población general. Actividades sencillas como mejorar los mecanismos de información para promover la consejería y la prueba voluntaria permitirían prevenir en gran parte nuevas infecciones en estos grupos. También es recomendable mantener una constante vigilancia epidemiológica en TS, considerando que si bien contribuyen poco a los casos incidentes, tienen una elevada incidencia y, por tanto, un constante riesgo de sufrir la infección y trasmitirla.

Una de las ventajas del modelo es su simplicidad, ya que usa datos de prevalencia existentes; ventaja que resalta si lo comparamos con otros métodos para obtener incidencia, como son los estudios de cohortes y el uso de pruebas de laboratorio para diagnosticar infecciones recientes. También el modelo puede aplicarse a las regiones del país, y así contribuir a la planificación regional. Adicionalmente, el modelo puede ser usado en la construcción de distintos escenarios bajo determinados supuestos, de modo que se pueda evaluar el impacto de posibles intervenciones en salud pública sobre la incidencia de la infección por $\mathrm{VIH}$. Finalmente, el modelo es perfectible y puede ser validado con la información recogida por los sistemas de vigilancia para el año estimado.

No obstante las bondades del MoT, no deja de tener limitaciones. Las proyecciones y extrapolaciones de casos de VIH/SIDA en general dependen de la disponibilidad de datos confiables, ideal que es difícil alcanzar sobre todo en el caso de los datos regionales. Esto se expresa, en este estudio, en los amplios rangos de incertidumbre hallados. Por ejemplo, muchos de los estudios consultados se realizaron en muestras pequeñas o poco representativas de los subgrupos respectivos; se observó, además, un importante subregistro de los casos de VIH/SIDA; tampoco se contó con prevalencias a nivel nacional ni datos actualizados, a falta de los cuales se emplearon fuentes que podrían tener sesgo de selección como la hoja de monitorización de los CERITSS. Lamentablemente, la información proporcionada por los sistemas de vigilancia también tiene sesgos relacionados con el acceso a las pruebas, el acceso al sistema de salud y a la variabilidad en el tiempo que transcurre desde que ocurre la infección hasta que se realiza el diagnóstico del $\mathrm{VIH}{ }^{(45)}$.
Además, el modelo no toma en cuenta diferentes niveles de riesgo dentro de un mismo grupo; tampoco toma en cuenta el traslape entre diferentes comportamientos de riesgo, fenómeno que puede tener un efecto importante sobre la dinámica de la epidemia. Otra limitación es la no inclusión de la proporción de personas viviendo con el VIH que reciben TARGA, y esto puede tener un impacto importante en la transmisión ${ }^{(46)}$. Finalmente, las estimaciones de incidencia son válidas solo para un año, siendo difícil repetir el cálculo de manera anual, tanto por la obtención de la información actualizada como por su sistematización para los fines del modelo. Un tiempo prudencial que se ha recomendado para las estimaciones es cinco años, pues hay que considerar que la dinámica de la infección y la composición de los grupos de riesgo pueden cambiar, sea por saturación o cambios de comportamiento.

El presente estudio representa la primera experiencia nacional de estimación de la incidencia para casos de VIH según modos de transmisión. Se presentaron diversas dificultades en la construcción del mismo, principalmente debido a la falta de datos completos; a pesar de ello, el modelo proporciona una estimación de la incidencia y su distribución entre los grupos de riesgo según el modo de transmisión, consistente con la distribución de los reportes de casos captados por el sistema de vigilancia. Las dificultades encontradas plantean la necesidad de implementar adecuados sistemas de vigilancia, que recoja los datos necesarios para hacer estimaciones más confiables. También es necesario desarrollar investigaciones puntuales que permitan contar con mejor información sobre la incidencia de $\mathrm{VIH}$ en el país. Finalmente, se debe estimular el tamiz en población masculina, donde se tiene menor información sobre la prevalencia de $\mathrm{VIH}$; al igual que continuar con actividades en población general como el tamizaje voluntario en MEF.

Agradecimientos: a ONUSIDA y DGE por la asistencia técnica.

Contribuciones de autoría: JOA, MP, LS, GR y PC participaron en la concepción del trabajo original. JOP, MP, CG, AW, LS y RT en la recolección de datos; JOA, MP, RT y AB participaron en la redacción del artículo. $A B$ realizó el análisis de incertidumbre. Todos los autores aprobaron la versión final del artículo.

Fuente de financiamiento: el trabajo fue coordinado a través de la oficina nacional del ONUSIDA, OPS, UNFPA, UNICEF y PNUD en colaboración con la Universidad Nacional Mayor de San Marcos y la DGE/MINSA.

Conflicto de intereses: los autores declaran no tener conflictos de interés. 


\section{REFERENCIAS BIBLIOGRÁFICAS}

1. Heaton LM, Komatsu R, Low-Beer D, Fowler TB, Way PO. Estimating the number of HIV infections averted: an approach and its issues. Sex Transm Infect. 2008;84(Suppl I):i92-6.

2. Gouws E. Methods for estimating HIV incidence [Internet]. UNAIDS quarterly update on HIV epidemiology/1Q 2010. Geneva: UNAIDS; 2010 [citado el 15 de septiembre del 2011]. Disponible en: https://www.unaids.org/en/media/ unaids/contentassets/dataimport/ pub/basedocument/2010/epi_ alert_1stqtr2010_en.pdf

3. Hallett TB, Zaba B, Todd J, Lopman B, Mwita W, Biraro S, et al. Estimating incidence from prevalence in generalised HIV epidemics: methods and validation. PLoS Med. 2008 Apr 8;5(4):e80.

4. Grassly NC, Morgan M, Walker N, Garnett G, Stanecki KA, Stover J, et al. Uncertainty in estimates of HIV/ AIDS: the estimation and application of plausibility bounds. Sex Transm Infect. 2004;80 Suppl 1:i31-38.

5. Chin J, Lwanga SK. Estimation and projection of adult AIDS cases: a simple epidemiological model. Bull World Health Organ. 1991;69(4):399406.

6. UNAIDS. Modeling the expected short-term distribution of new HIV infections by modes of transmission. Geneva: UNAIDS; 2012.

7. Pisani E, Garnett GP, Brown T, Stover J, Grassly NC, Hankins C, et al. Back to basics in HIV prevention: focus on exposure. BMJ. 2003;326(7403):13847.

8. Gouws E, White PJ, Stover J, Brown T. Short term estimates of adult HIV incidence by mode of transmission: Kenya and Thailand as examples. Sex Transm Infect. 2006;82 Suppl 3:iii5155.

9. Fleming DT, Wasserheit JN. From epidemiological synergy to publichealth policy and practice: the contribution of other sexually transmitted diseases to sexual transmission of HIV infection. Sex Transm Infect. 1999;75(1):3-17.

10. Gray RH, Wawer MJ, Brookmeyer R, Sewankambo NK, Serwadda D, Wabwire-Mangen F, et al. Probability of HIV-1 transmission per coital act in monogamous, heterosexual, HIV-1discordant couples in Rakai, Uganda. Lancet. 2001;357(9263):1149-53.

11. Gray RH, Li X, Kigozi G, Serwadda D, Nalugoda F, Watya $S$, et al. The impact of male circumcision on HIV incidence and cost per infection prevented: a stochastic simulation model from Rakai, Uganda. AIDS. 2007;21(7):845-50.

12. Instituto Nacional de Estadística e Informática. Perú: Encuesta Demográfica y de Salud Nacional. ENDES Continua 2004-2006. Informe Principal. Lima: INEI; 2007.

13. Instituto Nacional de Estadística e Informática. Perú: Encuesta Demográfica y de Salud Nacional. ENDES 2008. Línea Base INEI - MEF. Lima: INEI; 2008.

14. Instituto Nacional de Estadística e Informática. XI Censo de Población y VI de Vivienda 2007. Lima: INEI; 2007.

15. Comisión Nacional para el Desarrollo y Vida sin Drogas (DEVIDA). III Encuesta Nacional de Consumo de Drogas en Población General del Perú 2006. Lima: DEVIDA; 2008.

16. UNAIDS. Peru: HIV and AIDS estimates (2009) [Internet]. Geneva: UNAIDS; [citado el 15 de septiembre del 2011]. Disponible en: http:// www.unaids.org/en/regionscountries/ countries/peru/

17. Stover J, Bertrand JT, Shelton JD. Empirically based conversion factors for calculating couple-years of protection. Eval Rev. 2000;24(1):3-46.

18. Cárcamo CP, Campos PE, García PJ, Hughes JP, Garnett GP, Holmes KK, et al. Prevalences of sexually transmitted infections in young adults and female sex workers in Peru: a national population-based survey. Lancet Infect Dis. 2012;12(10):765-773.

19. Cáceres C, Konda K, Pecheny M, Chatterjee A, Lyerla R. Estimating the number of men who have sex with men in low and middle income countries. Sex Transm Infect. 2006;82 Suppl 3:iii3-9.

20. Dirección General de Epidemiología. Situación del VIH/SIDA en el Perú. Boletín Epidemiológico Mensual
[Internet]. 2009;(12) [citado el 15 de septiembre del 2011]. Disponible en: http://www.dge.gob.pe/vigilancia/ vih/boletines_vih.htm

21. Baral S, Sifakis F, Cleghorn F, Beyrer C. Elevated risk for HIV infection among men who have sex with men in lowand middle-income countries 20002006: a systematic review. PLoS Med. 2007;4(12):e339.

22. Lama J, Lucchetti A, Suarez L, LagunaTorres VA, Guanira JV, Pun M, et al. Association of herpes simplex virus type 2 infection and syphilis with human immunodeficiency virus infection among men who have sex with men in Peru. J Infect Dis. 2006;194(10):145966.

23. Clark JL, Caceres CF, Lescano AG, Konda KA, Leon SR, Jones FR, et al. Prevalence of same-sex behavior and associated characteristics among lowincome urban males in Peru. PLoS ONE. 2007;2(1):e778.

24. Sánchez J, Lama JR, Kusunoki L, Manrique H, Goicochea P, Lucchetti A, et al. HIV-1, sexually transmitted infections, and sexual behavior trends among men who have sex with men in Lima, Peru. J Acquir Immune Defic Syndr. 2007;44(5):578-85.

25. Cáceres CF, Konda K, Segura ER, Lyerla R. Epidemiology of male same-sex behaviour and associated sexual health indicators in low- and middle-income countries: 2003-2007 estimates. Sex Transm Infect. 2008;84 Suppl 1:i49-i56.

26. Pun M, Suárez L, Napanga E, Valdez W, Munayco C, Tirado J. Análisis de la situación epidemiológica del VIH/ SIDA en el Perú. Bases epidemiológicas para la prevención y control. Lima: Dirección General de Epidemiología/ MINSA; 2006.

27. Alarcon JO, Johnson KM, Courtois B, Rodriguez C, Sanchez J, Watts DM, et al. Determinants and prevalence of HIV infection in pregnant Peruvian women. AIDS. 2003;17(4):613-8.

28. Mathers BM, Degenhardt L, Phillips B, Wiessing L, Hickman M, Strathdee $\mathrm{SA}$, et al. Global epidemiology of injecting drug use and HIV among people who inject drugs: a systematic review. Lancet. 2008;372(9651):173345. 
29. Clark JL, Konda KA, Munayco CV, Pun M, Lescano AG, Leon SR, et al. Prevalence of HIV, herpes simplex virus-2, and syphilis in male sex partners of pregnant women in Peru. BMC Public Health. 2008;8:65.

30. NIMH Collaborative HIV/STD Prevention Trial Group. Sexually transmitted disease and HIV prevalence and risk factors in concentrated and generalized HIV epidemic settings. AIDS. 2007;21 Suppl 2:S81-90.

31. Ouellet L, Huo D, Bailey SL. HIV risk practices among needle exchange users and nonusers in Chicago. $J$ Acquir Immune Defic Syndr. 2004;37(1):1187-96.

32. Folch C, Meroño M, Casabona J. Factores asociados a la práctica de compartir jeringuillas usadas entre usuarios de droga por vía parenteral reclutados en la calle. Med Clin (Barc). 2006;127(14):526-32.

33. Ferreira AD, Caiaffa WT, Bastos FI, Mingoti SA; Projeto AjUDE-Brasil II. Profile of male Brazilian injecting drug users who have sex with men. Cad Saude Publica. 2006;22(4):849-60.

34. UNAIDS. Sex work and HIV. UNAIDS Technical Update. June 2002. Geneva: UNAIDS; 2002.

35. Montano S, Sanchez JL, Laguna-Torres A, Cuchi P, Avila MM, Weissenbacher $\mathrm{M}$, et al. Prevalences, genotypes and risk factors for HIV transmission in South America. J Acquir Immune Defic Syndr. 2005;40(1):57-64.

36. Sánchez J, Campos PE, Courtois B, Gutierrez L, Carrillo C, Alarcón J, et al. Prevention of sexually transmitted diseases (STDs) in female sex workers: prospective evaluation of condom promotion and strengthened STD services. Sex Transm Dis. 2003;30(4):273-9.

37. Trujillo L, Muñoz D, Gotuzzo E, Yi A, Watts DM. Sexual practices and prevalence of HIV, HTLV-I/ II, and Treponema pallidum among clandestine female sex workers in Lima, Peru. Sex Transm Dis. 1999;26(2):1158.

38. Miller GA, Mendoza W, Krone MR, Meza R, Cáceres CF, Coates TJ, et al. Clients of female sex workers in Lima, Peru. A bridge population for STD/HIV transmission? Sex Transm Dis. 2004;31(6):337-42.

39. Gutiérrez JP, Molina-Yépez D, Samuels F, Bertozzi SM. Uso inconsistente del condón entre trabajadoras sexuales en Ecuador: resultados de una encuesta de comportamientos. Salud pública de Méx. 2006;48(2):104-12.

40. Alarcón J. Determinants and prevalence of HIV and syphilis in pregnant women in Lima, Perú. Tesis de maestría University of Washington, DC: University of Washington; 1999.

41. UNAIDS. Incidence by Modes of Transmission [Internet]. Geneva: UNAIDS; [citado el 7 de junio del 2011]. Disponible en: http://www. unaids.org/en/dataanalysis/tools/ incidencebymodesoftransmission/

42. Thompson DA, Tsai YK, Gilman RH, Vivar A, Calderon M. Sexually transmitted diseases in a family planning and an antenatal clinic in Peru: limitations of current practices and analysis of the use of potential markers, $\mathrm{pH}$ testing, and Whiff testing. Sex Transm Dis. 2000;27(7):386-92.

43. Rivera JF, Roca O. La experiencia de Perú con un programa nacional de bancos de sangre. Rev Panam Salud Publica. 2003;13(2/3):165-71.

44. Sánchez J, Gotuzzo E, Escamilla J, Carrillo C, Phillips IA, Barrios C, et al. Gender differences in sexual practices and sexually transmitted infections among adults in Lima, Peru. Am J Public Health. 1996;86(8):1098-107.

45. Brancato G, Pezzoti P, Rapiti E, Perucci CA, Abeni D, Babbalacchio A, et al. Multiple imputation method for estimating incidence of HIV infection. 1997;26(5):1107-14.

46. UNSAIDS. Epidemiological Fact Sheet on HIV and AIDS: Core data on epidemiology and response [Internet]. Geneva: UNSAIDS; 2008 [citado el 15 julio 2011]. Disponible en: http://apps.who.int/globalatlas/ predefinedReports/EFS2008/full/ EFS2008_PE.pdf

Correspondencia: Jorge Alarcón

Dirección: Calle José Santos Chocano 199, Callao 2, Perú.

Teléfono: (511) 4525259

Correo electrónico:jalarconv@unmsm.edu.pe

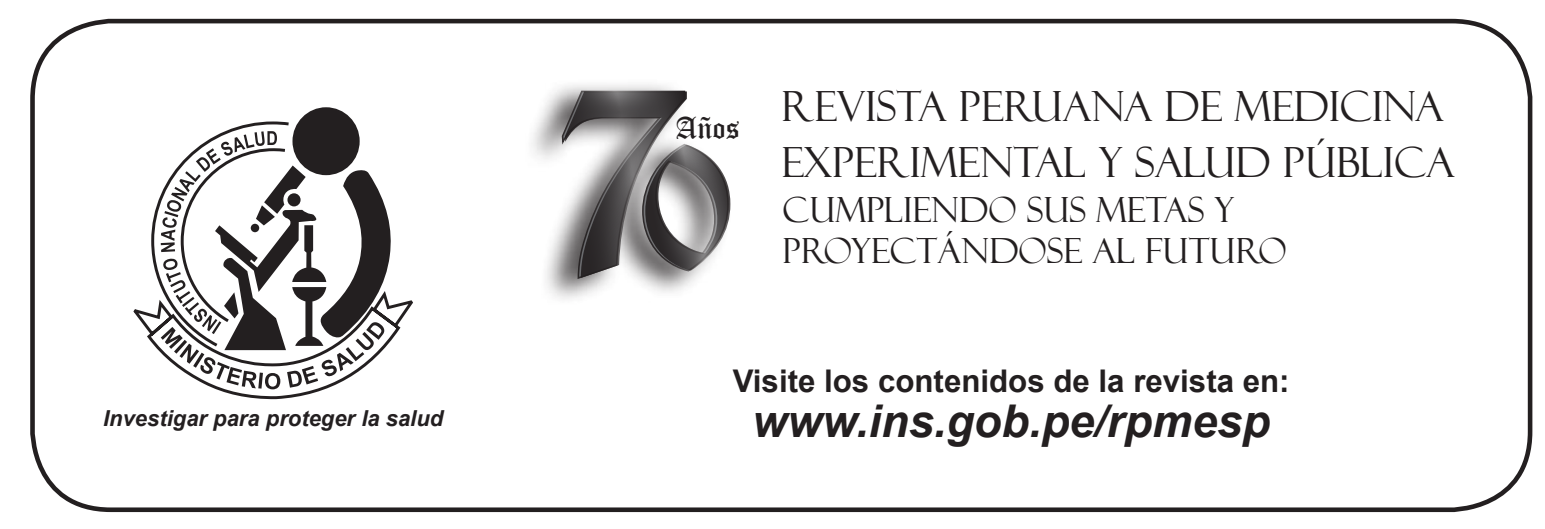

